\title{
Electromagnetic-field imbalance in surface plasmon polariton and its role in slow propagation and field- matter interaction
}

\author{
HIDEO IWASE ${ }^{1,2, *}$, TOSHIHIKO BABA ${ }^{1}$ \\ ${ }^{1}$ Department of Electrical and Computer Engineering, Yokohama National University, Yokohama 240-8501, Japan \\ ${ }^{2}$ Canon Inc., Tokyo 146-8501, Japan \\ *Corresponding author: iwase.hideo@mail.canon \\ Received XX Month XXXX; revised XX Month, XXXX; accepted XX Month XXXX; posted XX Month XXXX (Doc. ID XXXXX); published XX Month XXXX
}

We present the results of a theoretic study of the electromagnetic field imbalance in surface plasmon polaritons (SPPs), which reveal that the magnetic field components induced by the electric fields normal and parallel to a metal surface cancel each other in SPPs, resulting in an imbalance. A group velocity analysis shows that this imbalance contributes significantly to the slow propagation of SPPs. We also analyze the enhanced spontaneous emission and nonlinearity in plasmonic cavities, and the results indicate that the electromagnetic-field imbalance must be considered to correctly estimate the interaction strength.

OCIS codes: (240.6680) Surface plasmons; (260.3910) Metal optics; (270.5580) Quantum electrodynamics.

http://dx.doi.org/10.1364/AO.99.099999

\section{INTRODUCTION}

The electric field of surface plasmon polaritons (SPPs) exponentially decays with distance from a metal surface because they induce charge oscillation, which is known as a charge density wave (CDW), and is thus confined to a tiny modal cross-section beyond the diffraction limit [1,2] SPPs also exhibit slow propagation and a high density of states because of the strong dispersion of metal. Consequently, the SPP-emitter interaction is enhanced in a manner similar to that of a light emitter in a dielectric structure [3,4]. To date, the properties of SPPs have been investigated in various plasmonic structures, such as metal plates, periodic grooves (propagating SPP), particles (localized SPP) [5], and graphene-included structures [6], and enhanced spontaneous emission (SE) [7-16], Raman scattering [17,18], stimulated emission [19], singlephoton emission [20,21], and Rabi splitting in the strong-coupling regime [22] have been studied with the SPPs.

Recently, the imbalance between electric and magnetic fields of SPPs has attracted attention. Herein, we refer to this situation as EM imbalance. In our previous study on enhanced SE, the Purcell factor showing the enhancement fraction was expressed via the EMimbalance factor $\Theta$ (三 magnetic energy/electric energy), which approaches zero for SPPs on a metal plate [16]. Similar expressions were reported for different normalization conditions of the electric field [23], for localized SPPs [24], and for a dissipating rate [25]. Moreover, Johnson et al. investigated the EM imbalance in free plasma, which explained why it is caused by an increased kinetic energy of oscillating charges, resulting in the slow propagation of the electromagnetic field [26]. A very recent study on the EM imbalance in SPPs and surface phonon polaritons [27] showed that the magnetic energy can be quite small in a small metal sphere, which indicates that the degree of the imbalance depends on the size of a plasmonic cavity in which is confined theSPP or surface phonon polariton.

In this paper, we extend these studies to investigate the mechanism of extreme EM imbalance $(\Theta \approx 0)$ and of slow propagation of light over a metal plate. Moreover, we again discuss the Purcell factor as well as the enhanced nonlinearity to show that they are underestimated unless the EM imbalance is taken into account. In Sec. II, to clarify the mechanism, we show analytically and numerically the electromagnetic energy of SPPs and the kinetic energy of the CDW on a metal plate and in a metal sphere. In Sec. III, we discuss the slow propagation and analyze the group velocity by using Johnson's method. In Sec. IV, we analyze quantum mechanically the Purcell factor and compare the results with experimental results. Finally, we discuss the enhanced nonlinearity in a coupled plasmonic cavity [28], for which the EM imbalance exerts a much larger influence.

\section{MECHANISM OF ELECTROMAGNETIC IMBALANCE}

A. Surface plasmon polariton on a metal plate

Figure 1(a) shows a metal plate model in which SPPs with wave vector $\mathbf{k}$ travel along the metal-dielectric interface. The permittivity of the metal is approximated as $\varepsilon_{m}=\varepsilon_{\infty}-\varepsilon_{\infty} \omega_{p}^{2} / \omega^{2}$. The dielectric side has refractive index $n$, and its absorption is ignored. The electromagnetic fields $\mathbf{E}^{+}$and $\mathbf{B}^{+}\left(\propto \mathrm{e}^{i(\mathbf{k} \cdot \mathbf{x}+i \kappa z)-i \omega t}\right)$ of SPPs vary exponentially in the $z$ direction normal to the interface, as shown in Fig. $1(b)$. This is characterized by the imaginary propagation constant $i \kappa ; \kappa$ is negative on the metal side $(z<0)$ and positive on the dielectric side $(z$ $>0)[1,2]$. The CDW is characterized by the current density $\mathbf{J}^{+}=$ 
$i \varepsilon_{\infty} \varepsilon_{0}\left(\omega_{p}^{2} / \omega\right) \mathbf{E}^{+}$and charge density $\rho^{+}=-\int \nabla \cdot i \varepsilon_{\infty} \varepsilon_{0}\left(\omega_{p}^{2} / \omega\right) \mathbf{E}^{+} d t$ on the metal surface, where $\varepsilon_{0}$ is the permittivity in vacuum. We now present an example numerical calculation of the $\mathrm{CDW}$, assuming $n_{\mathrm{Au}}=$ $0.215+i 3.22$ at a wavelength $\lambda=612 \mathrm{~nm}$ on the metal $(\mathrm{Au})$ side and $n=$ 1.5 on the dielectric side [29]. In the Drude model, $n_{\mathrm{Au}}$ is characterized by the plasma frequency $\omega_{p}$ or the corresponding wavelength $\lambda_{p}=$ $2 \pi c / \omega_{p}=181 \mathrm{~nm}$ where $c$ is the speed of light in vacuum and $\varepsilon_{\infty}=1$. Figure 1 (c) shows the calculated distributions of $\operatorname{Re}\left(\mathrm{J}^{+}\right)$and $\operatorname{Re}\left(\rho^{+}\right)$of the CDW excited near the surface, where the SPPs propagate in the $x$ direction. Figure 1(d) illustrates the relation between the magnetic field $B_{y}\left(\equiv\left|\mathbf{B}^{+}\right|\right)$, the rotating electric field $E_{\mathrm{Fara}}$ induced through Faraday's law, the net parallel electric field $E_{x}\left(\equiv\left|\mathbf{E}^{+} \cdot \mathbf{k} / k\right|\right.$ where $\left.k \equiv|\mathbf{k}|\right)$, and the normal electric field $E_{z}\left(\equiv\left|\mathbf{E}^{+} \times \mathbf{k} / k\right|\right)$. The electric-field component $E_{x}$ is canceled by the superposition of $E_{\mathrm{Fara}}$ in a uniform medium, whereas it has nonzero values in SPPs and forms the CDW because of the rapid exponential decay in the $z$ direction.

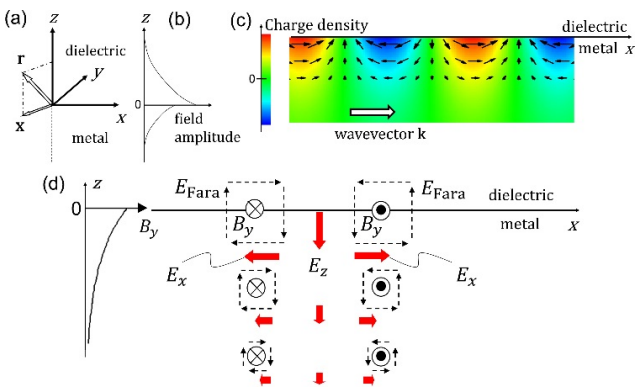

Fig. 1. (a) Uniform metal-dielectric interface in $(x, y)$ plane. Regions $z<0$ and $z>0$ are filled with metal (Au) and dielectric $(n=1.5)$, respectively. The vector $\mathbf{x}$ in the $(x, y)$ plane represents the projection of the position vector $\mathbf{r}$. (b) Field distribution of SPPs in $z$ direction. (c) Current density (arrows) and charge density (colors) of CDW near Au surface, when SPPs propagate in $x$ direction. (d) Schematic illustration showing how CDW is excited. $E_{\mathrm{Fara}}, E_{x}$ and $E_{z}$ are the rotating electric field induced by $B_{y}$ due to Faraday's law, the net parallel electric field, and the normal electric field, respectively.

The electric-field amplitudes $E_{x}$ and $E_{z}$ are obtained analytically by solving Maxwell's equations for $\mathbf{E}^{+}$, which are expressed as

$$
\begin{gathered}
\left(E_{x}, E_{z}\right) \propto\left(\frac{\kappa}{\varepsilon_{m}}, \frac{1}{\left|\varepsilon_{m}\right|}\right), \quad z<0, \\
\left(E_{x}, E_{z}\right) \propto\left(\frac{\kappa}{n^{2}}, \frac{1}{n^{2}}\right), \quad z>0,
\end{gathered}
$$

where $\kappa=-\left[k^{2}-\varepsilon_{m}(\omega / c)^{2}\right]^{1 / 2}$ at $\mathrm{z}<0, \kappa=\left[k^{2}-n^{2}(\omega / c)^{2}\right]^{1 / 2}$ at $\mathrm{z}>0$, and $\left[k^{2}-n^{2}(\omega / c)^{2}\right]^{1 / 2} / n^{2}+\left[k^{2}-\varepsilon_{m}(\omega / c)^{2}\right]^{1 / 2} / \varepsilon_{m}=$ 0 [2]. Maxwell's equation $\dot{\mathbf{B}}^{+}=\boldsymbol{\nabla} \times \mathbf{E}^{+}$also relates $B_{y}$ to $E_{x}$ and $E_{z}$ as follows:

$$
B_{y}=\frac{1}{\omega}|| k E_{z}|-| \kappa E_{x}|| .
$$

Equation (1c) shows that the magnetic field components induced by $E_{z}$ and $E_{x}$ partly cancel each other and $B_{y}$ decreases.

Figure 2(a) shows the distributions of electric-, magnetic-, and kinetic energy densities in the $z$ direction:

$$
\begin{gathered}
w_{\mathrm{E}}=\frac{\varepsilon_{\infty} \varepsilon_{0}\left|\mathbf{E}^{+}\right|^{2}}{2}(z<0), \quad \frac{n^{2} \varepsilon_{0}\left|\mathbf{E}^{+}\right|^{2}}{2}(z>0), \\
w_{\mathrm{B}}=\frac{\left|\mathbf{B}^{+}\right|^{2}}{2 \mu_{0}},
\end{gathered}
$$

$$
w_{\text {kin }}=\frac{\left|\mathbf{J}^{+}\right|^{2}}{2 \varepsilon_{\infty} \varepsilon_{0} \omega_{p}^{2}}
$$

and $\left|E_{x} / E_{z}\right|$ on the metal surface at different wavelengths $\lambda_{k}$ (三 $2 \pi / k)$ and frequencies $\omega$ normalized by the resonance frequency $\omega_{r}$ $\left[\equiv \omega_{p} /\left(\varepsilon_{\infty}+n^{2}\right)^{1 / 2}\right][1,2]$. The energies $\left(W_{\mathrm{E}}, W_{\mathrm{B}}, W_{\text {kin }}\right)$ are also shown in Fig. 2(b), which are given by the volume integrals of the energy densities. We also define the sum of the electric field and electric charge energies as $W_{\mathrm{EE}} \equiv W_{\mathrm{E}}+W_{\mathrm{kin}}$ and the total energy $W \equiv W_{\mathrm{E}}+W_{\mathrm{B}}+$ $W_{\text {kin. }}$ In this case,

$$
\Theta \equiv W_{\mathrm{B}} / W_{\mathrm{EE}}
$$

By using the relation $\varepsilon_{m}=\varepsilon_{\infty}-\varepsilon_{\infty} \omega_{p}^{2} / \omega^{2}, W_{\mathrm{EE}}$ may be written in the well-known form $\frac{1}{2} \int \varepsilon_{0} \tilde{\varepsilon}\left|\mathbf{E}^{+}\right|^{2} d \mathbf{r}$, where $\tilde{\varepsilon}=\partial\left(\varepsilon_{m} \omega\right) / \partial \omega$ in the metal and $\tilde{\varepsilon}=n^{2}$ in the dielectric $[30,31]$. Figure 2(b) shows that, as $\lambda_{k}$ becomes shorter $\left(\omega \rightarrow \omega_{r}\right), W_{\text {kin }}$ increases and $W_{\mathrm{B}}$ decreases while $W_{\mathrm{E}}$ does not change, and consequently $\Theta$ and $W_{\mathrm{EE}} / W$ approach zero and unity, respectively. Thus we obtain the relations

$$
\begin{gathered}
\frac{W_{\text {kin }}}{W}=\frac{1}{2}-\frac{W_{\mathrm{B}}}{W}=\frac{1}{2} \frac{1-\Theta}{1+\Theta}, \\
\frac{W_{\mathrm{E}}}{W}=\frac{1}{2} .
\end{gathered}
$$

As opposed to obtaining the same EM energies in a nondispersive dielectric medium, $W_{\mathrm{B}}<W_{\mathrm{EE}}$ and $\Theta<1$ because of the magnetic field cancelation.

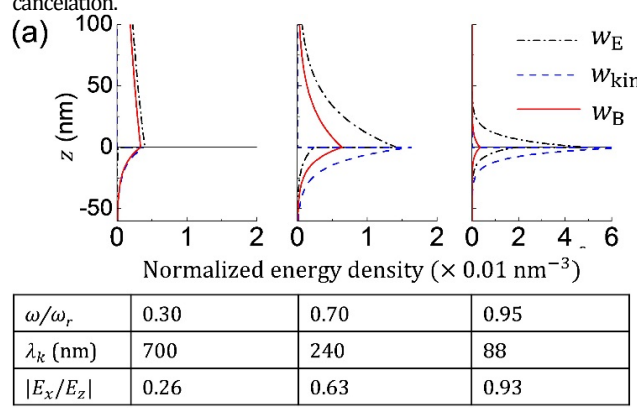

(b)

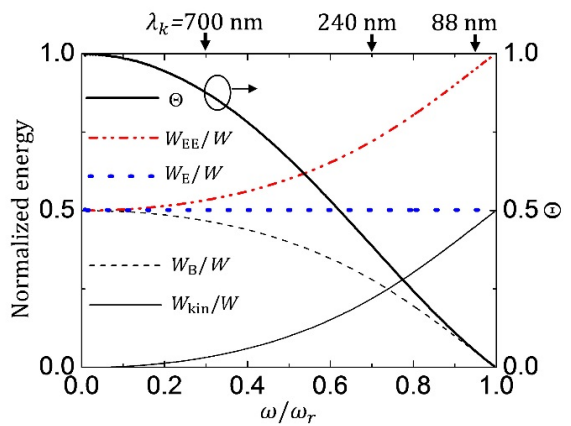

Fig. 2. (a) Calculated energy density distributions in $\mathrm{z}$ direction and $\omega / \omega_{r}, \lambda_{k}$, and $\left|E_{x} / E_{z}\right|$ at $z=+0$. (b) Normalized energies and $\Theta$, plotted as a function of $\omega / \omega_{r}$. Calculation model is the same as in Fig. 1.

B. Surface plasmon polariton around a metal sphere 
Previous work investigated the EM imbalance in a metal sphere [27]. We check the correspondence between SPPs on a metal plate and SPPs on a metal sphere, focusing on the field patterns and $\Theta$ for high-order SPP modes on the metal sphere Figure 3(a) shows the EM fields of the fundamental $(l=1)$ and higher-order $(l=2,3)$ modes excited around a 20 -nm-diameter Au sphere [32] (see Appendix A1 for analysis method). Standing waves of the modes have $2 l$ nodes and antinodes. Figure $3(\mathrm{~b})$ gives the wavelength of the standing wave, $\lambda_{s}$, mode volumes $V_{\text {mode }}[14,15]$, and $\Theta$. As $l$ increases, $\lambda_{s}$ decreases $\left(\omega \rightarrow \omega_{r}\right)$, and then $\Theta$ decreases in the same manner as the relation $\lambda_{k}-\Theta$ on a metal plate.

(a)

$I=1$

$\mid \mathbf{B}^{+}$
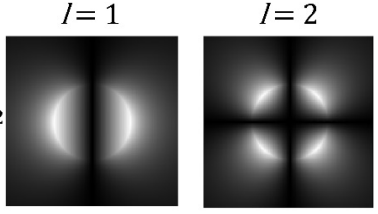

$l=3$

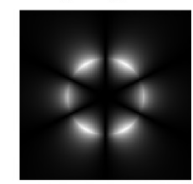

$\mid \mathbf{E}^{+}$
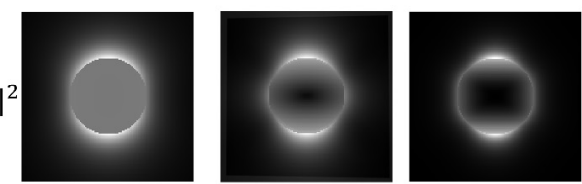

(b)

\begin{tabular}{|l|l|l|l|l|}
\cline { 3 - 5 } \multicolumn{2}{c|}{} & $l=1$ & $l=2$ & $I=3$ \\
\hline \multirow{3}{*}{$\begin{array}{c}2 r= \\
20 \mathrm{~nm}\end{array}$} & $\omega / \omega_{r}$ & 0.81 & 0.90 & $\sim 1.00$ \\
\cline { 2 - 5 } & $\Theta$ & 0.016 & 0.0058 & 0.0016 \\
\cline { 2 - 5 } & $V_{\text {rnode }}\left(\mathrm{nm}^{3}\right)$ & $5.9 \times 10^{3}$ & $3.0 \times 10^{3}$ & $1.5 \times 10^{3}$ \\
\cline { 2 - 5 } & $\lambda_{5}(\mathrm{~nm})$ & 63 & 31 & 21 \\
\hline \multirow{3}{*}{$\begin{array}{c}2 r= \\
50 \mathrm{~nm}\end{array}$} & $\omega / \omega_{r}$ & 0.75 & 0.87 & 0.99 \\
\cline { 2 - 5 } & $\Theta$ & 0.079 & 0.87 & 0.0099 \\
\cline { 2 - 5 } & $V_{\text {mode }}\left(\mathrm{nm}^{3}\right)$ & $7.7 \times 10^{4}$ & $4.4 \times 10^{4}$ & $2.5 \times 10^{4}$ \\
\cline { 2 - 5 } & $\lambda_{S}(\mathrm{~nm})$ & 157 & 78 & 52 \\
\hline
\end{tabular}

Fig. 3. (a) Distributions of $\left|\mathbf{B}^{+}\right|^{2}$ and $\left|\mathbf{E}^{+}\right|^{2}$ of SPPs with the mode number $l$ for SPP excited around a 20-nm-diameter Au sphere. (b) $\omega / \omega_{r}, \Theta, V_{\text {mode }}$, and $\lambda_{\mathrm{s}}$ for SPPs around 20- and 50-nm-diameter $\mathrm{Au}$ spheres.

\section{PROPAGATION OF SURFACE PLASMON POLARITON}

\section{A. Surface plasmon polariton on a metal plate}

According to a study by Johnson [26], the optical group velocity $v^{\mathrm{gr}, \mathrm{pl}}$ is interpreted as the modified phase velocity $v^{\mathrm{ph}, \mathrm{pl}}$ in a uniform free plasma (the superscript pl denotes a free plasma), which is multiplied by the ratio of the propagating energy density $w_{\mathrm{E}}^{\mathrm{pl}}+w_{\mathrm{B}}^{\mathrm{pl}}-w_{\text {kin }}^{\mathrm{pl}}$ to the total energy density $w_{\mathrm{E}}^{\mathrm{pl}}+w_{\mathrm{B}}^{\mathrm{pl}}+w_{\mathrm{kin}}^{\mathrm{pl}}$, giving

$$
v^{\mathrm{gr}, \mathrm{pl}}=\frac{w_{\mathrm{E}}^{\mathrm{pl}}+w_{\mathrm{B}}^{\mathrm{pl}}-w_{\mathrm{kin}}^{\mathrm{pl}}}{w_{\mathrm{E}}^{\mathrm{pl}}+w_{\mathrm{B}}^{\mathrm{pl}}+w_{\mathrm{kin}}^{\mathrm{p}}} v^{\mathrm{ph}, \mathrm{pl}},
$$

where $w_{\mathrm{E}}^{\mathrm{pl}}=w_{\mathrm{B}}^{\mathrm{pl}}+w_{\text {kin }}^{\mathrm{pl}}$ must hold. In Eq. (5), the propagating energy density is less than the total energy density by a factor $2 w_{\text {kin }}^{\mathrm{pl}}$ because the electrons do not propagate by themselves but their oscillation does. In addition, only the part of $w_{\mathrm{E}}^{\mathrm{pl}}$ that is equal to $w_{\mathrm{B}}^{\mathrm{pl}}$ can propagate. By applying this relation to SPPs on a metal plate, we obtain

$$
v^{\mathrm{gr}}=\frac{W-2 W_{\mathrm{kin}}}{W} v^{\mathrm{ph}}=\frac{2 \Theta}{1+\Theta} v^{\mathrm{ph}} .
$$

To verify Eq. (6), we calculate $2 \Theta /(1+\Theta)$ and $v^{\mathrm{gr}} / v^{\mathrm{ph}}$ independently by using the model of Fig. 1(a). Here, the former was obtained from the energies calculated in the same manner as used in Sec. II A, and the latter was evaluated from the $(\omega, k)$ dispersion relation as $(\partial \omega / \partial k) /(\omega / k)$. We confirm the relation $v^{\mathrm{gr}} / v^{\mathrm{ph}}=2 \Theta /(1+\Theta)$, as shown in Fig. 4. This means that the EM imbalance on a metal plate is essentially the same as that for a free plasma. Equation (6) indicates that an extremely low group velocity of the SPP occurs when $\Theta$ takes quite small values at frequencies close to $\omega_{r}$

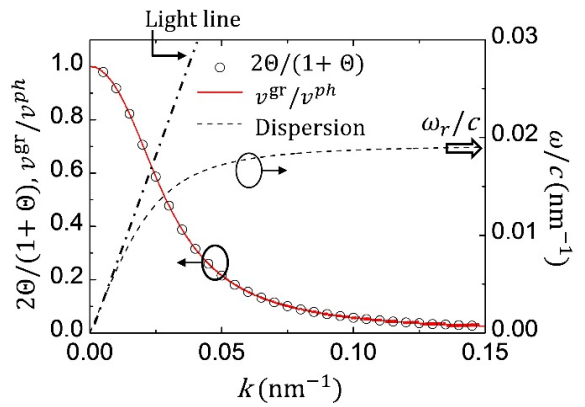

Fig. 4. $2 \Theta /(1+\Theta)$ (circles) and $v^{\mathrm{gr}} / v^{\mathrm{ph}}$ (solid line) for the SPP calculated with propagation constant $k$. The dashed line shows the SPP dispersion. The calculation model is the same as that used in Fig. 1.

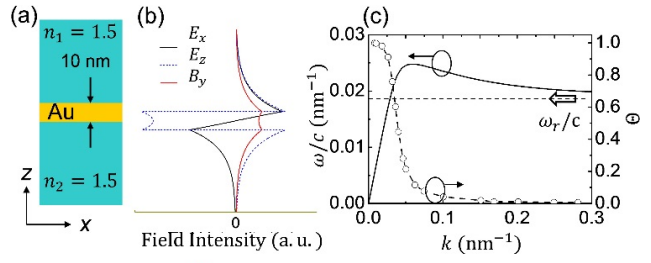

(d)

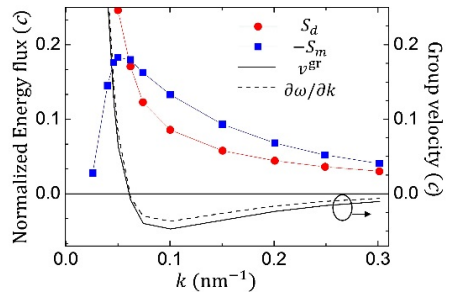

Fig. 5. (a) Thin Au film sandwiched by dielectric layers with a dielectric constant of 1.5. (b) Distributions of electric and magnetic fields of antisymmetric SPP mode. (c) Dispersion and $\Theta$ characteristics of SPP mode shown in panel (b). (d) Energy flux in Au (blue squares) and dielectric (red circles) normalized by energy density of field. The solid black line shows the group velocity $v^{\mathrm{gr}}\left(=S_{d}+S_{m}\right)$, and the dashed line shows $\partial \omega / \partial k$ estimated from the dispersion curve in panel (c). 
B. Surface plasmon polariton in a thin metal film

We now consider a thin metal film and its antisymmetric SPP mode, as shown in Fig. 5(a), which undergoes slow propagation over a wider range of $k$ than does a SPP on a metal plate $[1,16]$ To examine the EM imbalance, we simply calculate $\Theta$ and the energy flux in the $x$ direction: $s_{d}=\left(\mathbf{E}^{+} \times \mathbf{B}^{+}\right) \cdot \mathbf{k} / k$ in the dielectric and an analogous expression for $s_{m}$ in the metal (Au). Figure 5(d) shows the sum of the energy flux normalized by the energy density, $S_{d}=\iint s_{d} d y d z / \iint w d y d z$ and $S_{m}=$ $\iint s_{m} d y d z / \iint w d y d z$, where $\mathrm{w}=w_{\mathrm{E}}+w_{\mathrm{B}}+w_{\text {kin }}$ and the group velocity $v^{\mathrm{gr}}\left(=S_{d}+S_{m}\right)$, for the first antisymmetric SPP mode [1,2]; its fields, dispersion curve, and $\Theta$ are shown in Figs. 5(b) and (c). $S_{d}$ and $S_{m}$ have opposite signs because of the backward scattering of light by the conduction electrons in the metal. At $k<0.06 \mathrm{~nm}^{-1}$ in the plot of Fig. 5(d), $S_{d}$ decreases and $S_{m}$ increases as $k$ increases. They eventually cancel out at $k=0.06$ $\mathrm{nm}^{-1}$, where $v^{\mathrm{gr}}$ goes to zero. At $k>0.06 \mathrm{~nm}^{-1}$, both approach zero, which is attributed to the weakened magnetic field. In the thin metal layer, the cancelation of forward and backward energy fluxes and the decreases in the fluxes themselves result in slow light propagation over a wide range of $k$.

In an asymmetric structure $\left(n_{1}>n_{2}\right)$, since the propagation constant $k_{z}$ in the $z$ direction has a real part, the SPP transports its energy toward the dielectric with the larger index $n_{1}$, being coupled into the far filed (e.g., in the case of the KretschmannRaether (K-R) configuration). According to Ref. [33], the group velocity $\partial \omega / \partial k_{z}$ in the $z$ direction takes a quite small value at a frequency close to $\omega_{r}$, as $\partial \omega / \partial k$ does in Figs. 5(c) and (d). It indicates that the reduction of the magnetic field affects the energy transport in the $z$ direction and the far field coupling in the K-R configuration.

\section{FIELD-MATTER INTERACTION}

\section{A. Electric field enhancement by EM imbalance}

To determine how $\Theta$ affects the field-matter interaction, we first examine quantized electric field $\hat{\mathbf{E}}$ with $\Theta<1$, and the expectation value of the intensity operator $\hat{\mathbf{E}}^{2}$ with the Fock state $|N\rangle$. The Hamiltonian $H$ for a plasmonic cavity mode is generally expressed with the vector potential $\mathbf{A}(\mathbf{r}, t)$ as

$$
H=\frac{1}{2} \iiint\left[\frac{\partial[\varepsilon(\mathbf{r}) \omega]}{\partial \omega} \varepsilon_{0} \dot{\mathbf{A}}^{2}+\frac{(\nabla \times \mathbf{A})^{2}}{\mu_{0}}\right] d \mathbf{r},
$$

where $\dot{\mathbf{A}}=-\operatorname{Re}^{+}$. By introducing the normalized electric field $\mathbf{f}^{+}(\mathbf{r})$, satisfying $\iiint[\partial(\varepsilon \omega) / \partial \omega] \varepsilon_{0}\left|\mathbf{f}^{+}\right|^{2} d \mathbf{r}=1$ and $\mathbf{E}^{+}(\mathbf{r}, t)=$ $\mathrm{Df}^{+}(\mathbf{r}) e^{-i \omega t}$ where $D$ is the amplitude coefficient, $\mathbf{A}$ is expressed as

$$
\mathbf{A}(\mathbf{r}, t)=\frac{1}{2}\left[\frac{D}{i \omega} \mathbf{f}^{+}(\mathbf{r}) e^{-i \omega t}+\text { c. c. }\right],
$$

where c.c. is the complex conjugate of the first term. By using the mode volume $V_{\text {mode }} \equiv \iiint[\partial(\varepsilon \omega) / \partial \omega]\left|\mathbf{E}^{+}\right|^{2} d \mathrm{r} / \max \{[\partial(\varepsilon \omega) /$ $\left.\partial \omega]\left|\mathbf{E}^{+}\right|^{2}\right\}[3,14-16]$

$$
\mathbf{f}^{+}(\mathbf{r}) e^{-i \omega t}=\frac{\mathbf{E}^{+}(\mathbf{r}, t)}{\sqrt{V_{\operatorname{mode}^{\varepsilon_{0}} \max \left\{[\partial(\varepsilon \omega) / \partial \omega]\left|\mathbf{E}^{+}\right|^{2}\right\}}}}
$$

Next, $2 W_{\mathrm{EE}}=\iiint[\partial(\varepsilon \omega) / \partial \omega] \varepsilon_{0}\left|D \mathbf{f}^{+}\right|^{2} d \mathrm{r}=|D|^{2}$, and $2 W_{\mathrm{B}}=$ $|D|^{2} \iiint\left(1 / \mu_{0}\right)\left|\nabla \times\left(\mathbf{f}^{+} / i \omega\right)\right|^{2} d \mathbf{r}$. By introducing the parameter $\Theta$ $\equiv W_{\mathrm{B}} / W_{\mathrm{EE}}$, we obtain $1 /(1+\Theta)=W_{\mathrm{EE}} /\left(W_{\mathrm{EE}}+W_{\mathrm{B}}\right)$ and $\Theta /$ $(1+\Theta)=W_{\mathrm{B}} /\left(W_{\mathrm{EE}}+W_{\mathrm{B}}\right)$. For quantization, $D e^{-i \omega t}$ and $D e^{i \omega t}$ in Eq. (8) are replaced with the variables $p$ and $q$, which are defined as

$$
p(t)=\sqrt{1+\Theta} D \cos (\omega t)
$$

$$
q(t)=\sqrt{1+\Theta} D \sin (\omega t) / \omega .
$$

From Eqs. (7)-(10), we find that $H$ satisfies the following Hamilton equations: $H=\left(p^{2}+\omega^{2} q^{2}\right) / 2, \partial H / \partial p=p=\dot{q}$, and $\partial H / \partial q=\omega^{2} q=-\dot{p}$. After applying canonical field quantization, the classical variables $p$ and $q$ are identified with the quantum operators $\hat{Q}$ and $\hat{P}$, and introducing the annihilation operator $\hat{a}$ defined as $\hat{a} e^{-i \omega t} \equiv(\omega \hat{Q}+i \hat{P}) / \sqrt{2 \hbar \omega}$ and the creation operator $\hat{a}^{\dagger}$ defined as $\hat{a}^{\dagger} e^{i \omega t} \equiv(\omega \hat{Q}-i \hat{P}) / \sqrt{2 \hbar \omega}$, we obtain the following quantized fields [34]:

$$
\begin{gathered}
\hat{\mathbf{E}}(\mathbf{r}, \mathrm{t})=i \sqrt{\frac{\hbar \omega}{1+\Theta}} \mathbf{f}^{+}(\mathbf{r}) \hat{a} e^{-i \omega t}+\text { H.c., } \\
\widehat{\mathbf{B}}(\mathbf{r}, \mathrm{t})=\sqrt{\frac{\hbar}{(1+\Theta) \omega}} \nabla \times \mathbf{f}^{+}(\mathbf{r}) \hat{a} e^{-i \omega t}+\text { H.c., }
\end{gathered}
$$

where H.c. is the Hermitian conjugate of the first term

Using Eq. (11a), $\left\langle N\left|\hat{\mathbf{E}}^{2}\right| N\right\rangle$ is expressed as:

$$
\left\langle N\left|\hat{\mathbf{E}}^{2}\right| N\right\rangle=\frac{2}{1+\Theta} \frac{\hbar \omega}{\varepsilon_{0} \tilde{\varepsilon}}\left(N+\frac{1}{2}\right)\left\{\varepsilon_{0} \tilde{\varepsilon}\left|\mathbf{f}^{+}\right|^{2}\right\},
$$

in which $2 /(1+\Theta)\left(=2 W_{\mathrm{EE}} / W\right)$ shows the relative enhancement of the electric energy.

\section{B. Spontaneous emission enhancement by SPP}

To analyze the enhanced SE, we assume a two-level system, and the interaction between the emitter and SPP electric field is expressed as $\hat{V}=-\boldsymbol{\mu} \cdot \hat{\mathbf{E}}$, where $\boldsymbol{\mu}$ is the electric dipole near the metal surface. The emission rate into a single SPP mode at $\omega$ is $\Gamma^{\mathrm{SPP}}=\frac{2}{1+\Theta}(\pi \omega / \hbar)\left|\boldsymbol{\mu} \cdot \mathbf{f}^{+}\left(\mathbf{r}_{\mathrm{e}}\right)\right|^{2} S(\Omega, Q)$, where $\Omega$ and $\mathbf{r}_{\mathrm{e}}$ are the frequency and position of the dipole, respectively, and $S(\Omega, Q)$ is the Lorentzian spectrum with the $Q$ factor. The Purcell factor $F$ is $F \equiv \Gamma^{\mathrm{SPP}} / \gamma^{\mathrm{SE}}$, where $\gamma^{\mathrm{SE}}$ is the intrinsic SE rate $\left(=n \mu^{2} \Omega^{3} /\right.$ $\left.3 \pi \varepsilon_{0} \hbar c^{3}\right)$ in a bulk host material $[3,16,35]$. When $\left|\boldsymbol{\mu} \cdot \mathbf{f}^{+}\left(\mathbf{r}_{\mathrm{e}}\right)\right|=$ $\mu_{\max }\left|\mathbf{f}^{+}(\mathbf{r})\right|$ and $\omega=\Omega, F=\frac{2}{1+\Theta}\left(3 / 4 \pi^{2}\right)(\lambda / n)^{3}\left(Q / V_{\text {mode }}\right)$. For a dipole coupled to a SPP near the metal surface, $2 /(1+\Theta) \approx 2$, and $F$ becomes twice as large as the conventional value given by $F=\left(3 / 4 \pi^{2}\right)(\lambda / n)^{3}\left(Q / V_{\text {mode }}\right)[4]$. 


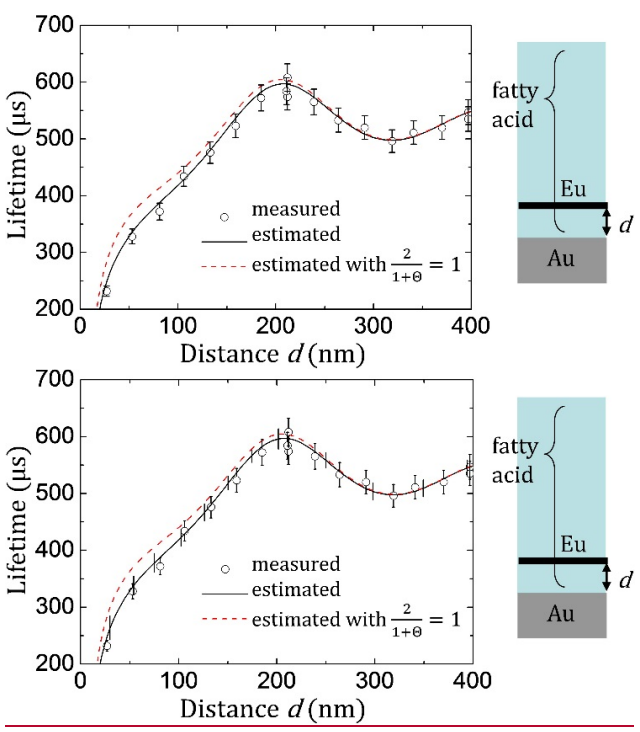

Fig. 6. Comparison between theoretically estimated lifetime with $2 /(1+\Theta)=1.60$ (solid line) and measured lifetime (circles) of optically excited Eu compound in Au-dielectric (fatty acid) layers, which were taken from Ref. [8]. The dashed line gives the theoretical values with $2 /(1+\Theta)=1$. The error bar for the measured lifetime is $\pm 4 \%$ $[8,10]$. The error bar for the theoretical lifetime (solid line) shows inaccuracies of $\gamma^{-1}$ and $\eta: \gamma_{\Delta}^{-1}=536 \pm 8 \mu$ s and $\eta=0.688 \pm 0.090$ The dashed line has the same fitting errors as shown by the error bars in the solid line.

Figure 6 shows the measured lifetime of a fluorescent material (Eu compound) lying in front of a Au plate [8], and the theoretical lifetime $\Gamma^{-1}\left(=\gamma^{-1}\left[\left(\bar{F}^{\mathrm{SPP}}+\bar{F}^{\text {non-SPP }}\right) \eta+(1-\right.\right.$ $\eta)]^{-1}$ ), where $\gamma$ and $\eta$ are the intrinsic decay rate of the fluorescent material without the metal plate and the quantum yield $\left(=\gamma^{\mathrm{SE}} / \gamma\right)$, respectively; $\gamma \bar{F}^{\mathrm{SPP}}$ is the emission rate into SPP modes and averaged over the randomly oriented emitters [16]; and $\gamma \bar{F}^{\text {non-SPP }}$ is the same but for non-SPP modes [36]. In this theoretical estimation, the SPP decay due to the absorption in $\mathrm{Au}$ was considered. The details of calculation and parameters for the theoretical estimate are summarized in Appendix A2. In Fig. 6 , the lifetime estimated without EM imbalance; namely, 2/(1+ $\Theta)=1$ (dashed line), slightly deviates from the measured lifetime (circles) with a $30 \%$ difference at most, whereas our estimate with the imbalance (solid line) fits much better.

\section{Nonlinearity enhancement by surface plasmon polariton}

This section shows that the effects of the EM imbalance increase in nonlinear multiphoton processes. We assume coupled plasmonic cavities and analyze the double resonance with nonlinear coupling coefficients $\beta_{1}$ and $\beta_{2}$ by using coupledmode theory [28]. For simplicity, we consider a nonlinearity only in a dielectric around the cavities. We first evaluate the frequency change $\delta \omega$ induced by a small change in $\varepsilon$ of the dielectric. In a plasmonic cavity, the electromagnetic field $\left(\mathbf{E}^{+}\right.$
$\mathbf{B}^{+}$) and electric polarization $\mathbf{P}^{+}$satisfy the following equation [37]:

$$
\begin{gathered}
\omega^{2} \iiint d \mathbf{r}\left(\left|\mathbf{B}^{+}\right|^{2} / \mu_{0}+\varepsilon \varepsilon_{0}\left|\mathbf{E}^{+}\right|^{2}+\frac{\vartheta(\mathbf{r})}{\varepsilon \varepsilon_{0} \omega_{p}^{2}}\left|\dot{\mathbf{P}}^{+}\right|^{2}\right) \\
=\iiint d \mathbf{r}\left\{\begin{array}{c}
\frac{1}{\mu_{0}}\left|\nabla \times \mathbf{E}^{+}\right|^{2}+\frac{1}{\varepsilon \varepsilon_{0} \mu_{0}^{2}}\left|\nabla \times \mathbf{B}^{+}\right|^{2} \\
\left.+\frac{\vartheta(\mathbf{r})}{\varepsilon \varepsilon_{0}}\left[\begin{array}{c}
\left|\dot{\mathbf{P}}^{+}\right|^{2}-\frac{1}{\mu_{0}}\left(\nabla \times \mathbf{B}^{+}\right) \cdot \dot{\mathbf{P}}^{-} \\
-\frac{1}{\mu_{0}}\left(\nabla \times \mathbf{B}^{-}\right) \cdot \dot{\mathbf{P}}^{+} \\
+\vartheta(\mathbf{r}) \varepsilon_{\infty} \varepsilon_{0} \omega_{p}^{2}\left|\mathbf{E}^{+}\right|^{2}
\end{array}\right]\right\},
\end{array}\right.
\end{gathered}
$$

where $\varepsilon=\varepsilon_{\infty}=1$ and $\vartheta(\mathbf{r})=1$ in a metal, $\varepsilon=n^{2}$ and $\vartheta(\mathbf{r})=0$ in a dielectric, and the superscript "-" is the complex conjugate of the same with "+." Consider a small change $\varepsilon \rightarrow \varepsilon+\delta \varepsilon$ and $\omega \rightarrow \omega+\delta \omega$; in a first-order approximation [38], we obtain

$$
\frac{\delta \omega}{\omega}=-\frac{\int_{\text {in dielectric }} \delta \varepsilon \varepsilon_{0}\left|\mathbf{E}^{+}\right|^{2} d \mathbf{r}}{\int\left(\frac{1}{\mu_{0}}\left|\mathbf{B}^{+}\right|^{2}+\varepsilon \varepsilon_{0}\left|\mathbf{E}^{+}\right|^{2}+\frac{\vartheta(\mathbf{r})}{\varepsilon \varepsilon_{0} \omega_{p}^{2}}\left|\dot{\mathbf{P}}^{+}\right|^{2}\right) d \mathbf{r}} .
$$

By using $\Theta$, which is

$$
\Theta=\frac{\int\left|\mathbf{B}^{+}\right|^{2} / \mu_{0} d \mathbf{r}}{\int\left(\varepsilon \varepsilon_{0}\left|\mathbf{E}^{+}\right|^{2}+\frac{\vartheta(\mathbf{r})}{\varepsilon \varepsilon_{0} \omega_{p}^{2}}\left|\dot{\mathbf{P}}^{+}\right|^{2}\right) d \mathbf{r}},
$$

and $\varepsilon_{0}\left|\mathbf{E}^{+}\right|^{2}+\left|\dot{\mathbf{P}}^{+}\right|^{2} / \varepsilon \varepsilon_{0} \omega_{p}^{2}=\varepsilon_{0}[\partial(\omega \varepsilon) / \partial \omega]\left|\mathbf{E}^{+}\right|^{2}$, we obtain

$$
\frac{\delta \omega}{\omega}=-\frac{2}{1+\Theta} \frac{1}{2} \frac{\int_{\text {in dielectric } \delta \varepsilon \varepsilon_{0}\left|\mathbf{E}^{+}\right|^{2} d \mathbf{r}}}{\int \varepsilon_{0}[\partial(\omega \varepsilon) / \partial \omega]\left|\mathbf{E}^{+}\right|^{2} d \mathbf{r}},
$$

where the right-hand side is multiplied by a factor $2 /(1+\Theta)$ in comparison with the conventional formula [38]

In accordance with Ref. [28] (also see Appendix A3), by introducing the optical susceptibility $\chi_{i j k}$ in the dielectric and relating $\delta \varepsilon \mathbf{E}^{+}$to the electric polarization induced by $\chi_{i j k}, \beta_{1}$ and $\beta_{2}$ are expressed as

$$
\begin{aligned}
& \beta_{1}=\frac{1}{4}\left(\frac{2}{1+\Theta_{1}}\right)^{2}\left(\frac{2}{1+\Theta_{2}}\right)^{1 / 2} \\
& \sum_{i, j, k}^{x, y, z} \int \varepsilon \varepsilon_{0} \chi_{i j k}\left[u_{1, i}^{-}\left(u_{1, j}^{-} u_{2, k}^{+}+u_{2, j}^{+} u_{1, k}^{-}\right)\right] d \boldsymbol{r} \\
& \beta_{2}=\frac{1}{4}\left(\frac{2}{1+\Theta_{1}}\right)\left(\frac{2}{1+\Theta_{2}}\right)^{3 / 2} \\
& \sum_{i, j, k}^{x, y, z} \int \varepsilon \varepsilon_{0} \chi_{i j k} u_{2, i}^{-} u_{1, j}^{+} u_{1, k}^{+} d \boldsymbol{r}
\end{aligned}
$$

where $\mathbf{u}_{l}^{+}\left[=\left(u_{l, x}^{+}, u_{l, y}^{+}, u_{l, z}^{+}\right)\right]$is the normalized electric field of mode $l(l=1,2)$ and is defined as $\mathbf{u}_{l}^{+}=\mathbf{E}_{l}^{+} /\left[\int \varepsilon_{0} \tilde{\varepsilon}\left|\mathbf{E}_{l}^{+}\right|^{2} d \mathbf{r}\right]^{1 / 2}$. Note that $\mathbf{u}_{l}^{-}$is the complex conjugate of $\mathbf{u}_{l}^{+} . \Theta_{l}$ is that for $\mathbf{u}_{l}^{+}$. As $\Theta_{l}$ approaches unity, the term $\sqrt{2 /\left(1+\Theta_{l}\right)}$ also approaches unity, and Eqs. (17a) and (17b) give the known formulas without the EM imbalance [28].

(a)

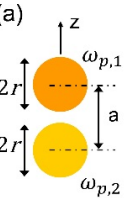

$(\mathrm{b})$
\begin{tabular}{|l|l|l|}
\hline$(i, j, k)$ & $\operatorname{Re} \beta_{1} / \varepsilon \varepsilon_{0}^{-\frac{1}{2}} \chi_{i j k}$ & $\operatorname{Re} \beta_{2} / \varepsilon \varepsilon_{0}^{-\frac{1}{2}} \chi_{i j k}$ \\
\hline$(z, z, z)$ & $57(10)$ & $28(5.1)$ \\
\hline$(z, x, x),(z, y, y)$ & $-10(-1.9)$ & $13(2.5)$ \\
\hline $\begin{array}{l}(x, x, z),(x, z, x) \\
(y, y, z],(y, z, y)\end{array}$ & $8.5(1.6)$ & $-5.1(-0.9)$ \\
\hline
\end{tabular}

\begin{tabular}{|l|}
\hline 書式変更: フォントの色：自動 \\
\hline 書式変更：フォントの色：自動 \\
\hline 書式変更：フォントの色：自動 \\
\hline 書式変更：フォントの色：自動 \\
\hline
\end{tabular}


Fig. 7. (a) Schematic of two conductive spheres with different plasma frequencies $\omega_{p, 1}$ and $\omega_{p, 2}\left(=2 \omega_{p, 1}\right.$ ). (b) Calculated values for Eqs. (17a) and (17b) for doubly resonantSPP modes in the structure of panel (a). The imaginary part (not shown) is more than ten times smaller than the real part. Terms with other $(i, j, k)$ go to zero. Values in parentheses were estimated by using the formula from Ref. [28].

Figure 7 (a) shows a model of two metal spheres with diameter $2 r$ and the center distance $a$ set at $0.10 \times 2 \pi c / \omega_{p, 1}$ and $0.12 \times 2 \pi c / \omega_{p, 1}$, and different plasma frequencies $\omega_{p, 1}$ and $\omega_{p, 2}=2 \omega_{p, 1}$ [39]. Figure 7(b) shows the results of Eqs. (17a) and $(17 \mathrm{~b})$ calculated for SPPs excited around this model. The values estimated by using the formulas without EM imbalance [28] are shown in parentheses. The values with EM imbalance are almost six-times greater than those without EM imbalance.

\section{CONCLUSION}

This study reveals that the magnetic field components in SPPs induced by electric fields normal and parallel to a metal surface partly cancel each other, resulting in EM imbalance and slow propagation of the SPPs. This knowledge is valuable for understanding the light propagation in plasmonic waveguides. In the theoretical analysis of the Purcell factor near a plasmonic structure and of the enhanced nonlinearity in coupled plasmonic cavities, we show that the EM imbalance must be considered to avoid underestimating the interaction Although we studied only two cases of field-matter interactions, the EM imbalance should have several times larger effects in any multi-photon interactions.

We would finally suggest three studies of interest, relevant to the EM imbalance. The first one is that the electric field enhancement and slow propagation can increase the sensitivity in sensing; dielectric and metalincluded multilayers have been compared for sensing applications [40]. The enhanced nonlinearities can increase the sensitivity in SPPenhanced Raman scattering and other nonlinear sensing, although they have not been discussed, yet. The second one is regarding assembled SPP nanocavities. The energy exchange between neighboring cavities may be decreased by the reduced magnetic field. Since that energy exchange deviates their resonance frequencies [41], the modification of the EM imbalance can be a scheme to design assembled cavities. The third one is the further investigation of SPP's radiative decay rate. SPP modes in a metal sphere have small radiative decay rates [32]. Only low energy dipole or quadrupole modes contribute to the far field radiation in the SPP excited around a metal nanotip [42]. We expect the small energy flux induced by the reduced magnetic field to be related to such small radiative decay rates.

\section{Appendices}

A1. SPP around a small metal sphere

Electromagnetic fields around a metal sphere have been theoretically analyzed in the spherical coordinates $(r, \theta, \varphi)$ [43] For SPPs, connecting the electromagnetic fields inside and outside a metal sphere with radius $\mathrm{R}$, and with the boundary conditions of $B_{l, \varphi}^{+}$and $E_{l, r}^{+}$, we obtain

$$
\begin{gathered}
B_{l, \varphi}^{+}=h_{l}^{(1)}(k \mathrm{R}) j_{l}\left(k_{m} r\right) \frac{d}{d \theta} P_{l}(\cos \theta), \\
E_{l, r}^{+}=-i \frac{\omega}{k_{m}^{2}} l(l+1) \frac{h_{l}^{(1)}(k \mathrm{R}) j_{l}\left(k_{m} r\right)}{r} P_{l}(\cos \theta), \\
E_{l, \theta}^{+}=-i \frac{\omega}{k_{m}^{2}} \frac{1}{r} h_{l}^{(1)}(k \mathrm{R}) \frac{d}{d r}\left[r j_{l}\left(k_{m} r\right)\right] \frac{d}{d \theta} P_{l}(\cos \theta),
\end{gathered}
$$

inside the metal $(r<\mathrm{R})$, and

$$
\begin{gathered}
B_{l, \varphi}^{+}=h_{l}^{(1)}(k r) j_{l}\left(k_{m} \mathrm{R}\right) \frac{d}{d \theta} P_{l}(\cos \theta), \\
E_{l, r}^{+}=-i \frac{\omega}{k^{2}} l(l+1) \frac{h_{l}^{(1)}(k r) j_{l}\left(k_{m} \mathrm{R}\right)}{r} P_{l}(\cos \theta), \\
E_{l, \theta}^{+}=-i \frac{\omega}{k^{2}} \frac{1}{r} \frac{d}{d r}\left[r h_{l}^{(1)}(k r)\right] j_{l}\left(k_{m} \mathrm{R}\right) \frac{d}{d \theta} P_{l}(\cos \theta),
\end{gathered}
$$

outside of the metal $(r>\mathrm{R})$, where $k^{2}=n^{2} \mu \omega^{2}$ and $k_{m}^{2}=$ $\varepsilon_{m} \mu \omega^{2}$. The functions $h_{l}^{(1)}(x), j_{l}(x)$, and $P_{l}(x)$ are the first spherical Huankel function, first spherical Bessel function, and Legendre polynomial, respectively. The frequency of mode $l$ is obtained by solving the boundary condition of $\operatorname{Re}\left(E_{l, \theta}^{+}\right)$at $r=\mathrm{R}$ neglecting the small discontinuity of $\operatorname{Im}\left(E_{l, \theta}^{+}\right) \cdot \operatorname{Im}\left(E_{l, \theta}^{+}\right) / \operatorname{Re}\left(E_{l, \theta}^{+}\right)$ is less than 0.003 for a Au sphere with $\mathrm{R}<10 \mathrm{~nm}$.

\section{A2. Fluorescence lifetime of material lying in front of a metal plate}

Considering the relaxation of an excited fluorescent material through emission into SPP and non-SPP modes, and through nonradiative processes, the lifetime $\Gamma^{-1}$ of the emitter is

$$
\Gamma^{-1}=\gamma^{-1} \times\left[\left(\bar{F}^{\mathrm{SPP}}+\bar{F}^{\text {non-SPP }}\right) \eta+(1-\eta)\right]^{-1}
$$

where $\gamma^{-1}$ is the intrinsic lifetime of the fluorescent material in its bulk host material, and $\eta$ is its quantum yield. $\bar{F}^{\text {SPP }}$ and $\bar{F}^{\text {non-SPP }}$ are Purcell factors for the SPP and non-SPP modes, respectively. For randomly oriented electric dipoles in a fluorescent material, they are

$$
\begin{gathered}
\bar{F}^{\mathrm{SPP}}=\frac{1}{3} F_{\perp}^{\mathrm{SPP}}+\frac{2}{3} F_{\|}^{\mathrm{SPP}}, \\
\bar{F}^{\mathrm{non}-\mathrm{SPP}}=\frac{1}{3} F_{p, \perp}+\frac{2}{3}\left(F_{s, \|}+F_{p, \|}\right),
\end{gathered}
$$

where the subscripts $\perp$ and $\|$ represent the cases in which the dipole points normal and perpendicular to the metal surface, respectively, and the subscripts $s$ and $p$ represent the cases in which the emission is $s$ and $p$ polarized, respectively. The equations for $F_{\perp}^{\mathrm{SPP}}, F_{\|}^{\mathrm{SPP}}$, and for $F_{p, \perp}, F_{s, \|}$, and $F_{p, \|}$ are available in Refs. [16] and [36], respectively; in Ref. [36], emission of an emitter sandwiched by two reflective interfaces is analyzed. SPP's dispersion and field patterns for calculating $F_{\perp}^{\text {SPP }}$ and $F_{\|}^{\mathrm{SPP}}$ were obtained in the same manner as that in Section 2, and the $\mathrm{Q}$ factor was obtained by solving the complex dispersion equation [16]. In the calculation of $F_{p, \perp}, F_{s, \|}$, and $F_{p, \|}$, we assumed a Au/dielectric interface and the emission into dielectric modes above the light line $(\omega>c k / n)$ was considered. The parameter values for estimating $\Gamma^{-1}$ in Fig. 6 were taken from Refs. [10,29]: $n_{\mathrm{Au}}=0.215+i 3.22$ for the $\mathrm{Eu}^{3+}$ emission wavelength of $\lambda=612 \mathrm{~nm}$ (equivalently, $\lambda_{p}=181 \mathrm{~nm}, \varepsilon_{\infty}=1$, and $c / \gamma_{\text {damp }}=797 \mathrm{~nm}$ where $\gamma_{\text {damp }}$ is the damping rate of light), and $n=1.5$ for the dielectric material. For the fluorescent material $\gamma^{-1}=536 \pm 8 \mu$ s and $\eta=0.688_{\Delta} \pm 0.090 \gamma^{=1}=536 \mu \mathrm{s}$ and $\eta=0.688$. which were estimated by fitting the measured lifetimes at $d>200 \mathrm{~nm}$, where emission into non-SPP modes is dominant without influence of the factor $2 /(1+\Theta)$ : The value of $2 /(1+\Theta)$ is theoretically estimated asby the formula presented in Sec. II; $2 /(1+\Theta)=1.60$.

The lifetime measurement of $\mathrm{Eu}^{3+}$ compound on a fatty acid/mirror structure was conducted by K. H. Drexhage [8,9] and by $\mathrm{H}$. Kuhn, et al. [7], for several types of mirrors, including $\mathrm{Au}$, $\mathrm{Ag}$, and $\mathrm{Cu}$ plates, and a fatty acid/air interface. In their studies, the fatty acid layers were deposited by using Langmuir-Blodgett technique, their thickness achieved within an accuracy of a few tenths of a percent [9]. They estimated that the inaccuracy of the

\begin{tabular}{|l|l|}
\hline 書式変更: フォント : +見出しのフォント (Cambria) \\
\hline 書式変更: フォント : $9 \mathrm{pt}$ \\
\hline 書式変更：フォント : +見出しのフォント (Cambria) \\
\hline 書式変更：フォント : +見出しのフォント (Cambria), 斜体 \\
\hline 書式変更：フォント : $9 \mathrm{pt}$ \\
\hline
\end{tabular}


measured lifetimes was within 3-4\% [10]. Details of the measurement and fluorescence decay curve can be found in Ref. [44]. The measured lifetime was theoretically analyzed in the classical electrodynamics theory by R. R. Chance, W. L. Barns and their coworkers [10,11]; the measured lifetime well agreed with the theoretical one with parameters $\gamma^{-1}$ and $\eta$, and random dipolar orientation. It should be noted that in their analysis (the classical electrodynamics analysis, in which the lifetime which was wasnumerically estimated throughfrom a decay rate of athe classical electric dipole moment; induced by the reflection from a metal plate, the estimated lifetime numerically includes the effect of-emission enhancement effect due to EM imbalance, but its effect cannot be separated from other enhancement effects by their analysis [16]. = In ourthis study, we used the quantum analysis, in which the emission enhancement due to EMimbalance is explicitly expressed asby the factor $2 /(1+\Theta)$. In our quantum analysis, tThehe experimental lifetime in Fig. 6 was taken from Drexhage's work [8], and the values of $\gamma^{-1}$ and $\eta$ for them were taken from Chance's work [10].

\section{A3. Deviations of $\boldsymbol{\beta}_{1}$ and $\boldsymbol{\beta}_{2}$ for coupled modes}

Relating $\delta \varepsilon\left|\mathbf{E}^{+}\right|^{2}=\left(\mathbf{E}^{-} \cdot \delta \mathbf{P}^{+}+\mathbf{E}^{+} \cdot \delta \mathbf{P}^{-}\right) / 2$ in Eq. (16) to the nonlinear polarization $\delta P_{i}^{+}=\sum_{j, k}^{x, y, z} \varepsilon_{0} \varepsilon \chi_{i j k} E_{j}^{+} E_{k}^{+}(i, j, k=$ $\{x, y, z\})$ induced by $\mathbf{E}^{+}=\operatorname{Re}\left[\mathbf{E}_{1}^{+} e^{-i \omega_{1} t}+\mathbf{E}_{2}^{+} e^{-i \omega_{2} t}\right]$ where $\mathbf{E}_{l}^{+}$is the electric field of mode $l$, and leaving only the terms satisfying $\omega_{2}=2 \omega_{1}$, we obtain

$$
\frac{\delta \omega_{2}}{\omega_{2}}=-\frac{2}{1+\Theta_{2}} \frac{1}{4} \frac{\left[\int_{\text {in dielectric }} \sum_{i j k} \varepsilon_{0} \varepsilon \chi_{i j k} E_{2, i}^{-} E_{1, j}^{+} E_{1, k}^{+} d \mathbf{r}+\mathrm{CC}\right]}{\int \varepsilon_{0}[\partial(\omega \varepsilon) / \partial \omega]\left|\mathbf{E}_{2}^{+}\right|^{2} d \mathbf{r}} .
$$

Equation (17b) is derived by comparing Eq. (22) to the corresponding formula for dielectric cavity modes in Ref. [28] which we multiplied by $\sqrt{2 /\left(1+\Theta_{l}\right)}(l=1,2)$ so that $\left|a_{l}\right|^{2}$ represents the electromagnetic energy of mode $l$. Note that $[(1+$ $\left.\left.\Theta_{l}\right) / 2\right] \int \varepsilon_{0}[\partial(\omega \varepsilon) / \partial \omega]\left|\mathbf{E}_{l}^{+}\right|^{2} d \mathbf{r}$ is the electromagnetic energy. $\beta_{1}$ in Eq. (17a) is derived in the same manner.

\section{References}

1. H. Raether, Surface Plasmons on Smooth and Rough Surfaces and on Gratings (Springer, Berlin, 1988)

2. E. N. Economou, "Surface Plasmons in Thin Films," Phys. Rev. 182, 539 (1969).

3. E. A. Hinds, "Perturbative cavity quantum electrodynamics," in Cavity Quantum Electrodynamics, P. R. Berman, ed. (Academic, New York, 1994).

4. K. J. Vahala, "Optical microcavities," Nature 424, 839 (2003)

5. W. A. Murray, and W. L. Barnes, "Plasmonic Materials," Advanced Materials 19, 3771 (2007)

6. T. J. Constant, S. M. Hornett, D. E. Chang and E. Hendry, "All-optical generation of surface plasmons in graphene," Nature Physics 12, 124 (2016).

7. H. Kuhn, "Classical Aspects of Energy Transfer in Molecular Systems," J. Chem. Phys. 53, 101 (1970).

8. K. H. Drexhage, "Interaction of light with monomolecular dye lasers," in Progress in Optics XII, E. Wolf, ed. (North-Holland, Amsterdam, 1974).

9. K. H. Drexhage, "Monomolecular laser and light," Sci. Am., 222, 108 (1970)

10. R. R. Chance, A. Prock, and R. Silbey, "Molecular Fluorescence and Energy Transfer Near Interfaces," Adv. Chem. Phys. 37, 1 (1978).

11. W. L. Barnes, "Fluorescence near interfaces: The role of photonic mode density,"J. Mod. Opt. 45, 661 (1998).

12. I. Gontijo, M. Boroditsky, E. Yablonovitch, S. Keller, U. K. Mishra, and S. P. DenBaars, "Coupling of InGaN quantum-well photoluminescence to silver surface plasmons," Phys. Rev. B 60, 11564 (1999).
13. K. Okamoto, I. Niki, A. Shvartser, Y. Narukawa, T. Mukai and A. Scherer, "Surface-plasmon-enhanced light emitters based on InGaN quantum wells," Nature Materials 3, 601 (2004)

14. J. Vučković, M. Loncar and A. Scherer, "Surface plasmon enhanced lightemitting diode," IEEE J. Quantum Electron. 36, 1131 (2000).

15. H. Iwase, D. Englund, and J. Vučković, "Spontaneous emission control in high-extraction efficiency plasmonic crystals," Opt. Express 16, 426 (2008).

16. H. Iwase, D. Englund, and J. Vučković, "Analysis of the Purcell effect in photonic and plasmonic crystals with losses," Opt. Express, 18, 16546 (2010)

17. M. Khorasaninejad, S. M. Raeis-Zadeh, S. Jafarlou, M. J. Wesolowski, C. R. Daley, J. B. Flannery, J. Forrest, S. Safavi-Naeini, and S. S. Sainib, “Highly Enhanced Raman Scattering of Graphene using Plasmonic NanoStructure," Sci. Rep. 3, 2936 (2013).

18. Frontiers of Surface-Enhanced Raman Scattering: Single Nanoparticle and Single Cells, Y. Ozaki, K. Kneipp, and R. Aroca, ed. (Wiley, United Kingdom, 2014).

19. F. van Beijnum, P. J. van Veldhoven, E. J. Geluk, M. J. A. de Dood, G. W.' Hooft, and M. P. van Exter, "Surface Plasmon Lasing Observed in Metal Hole Arrays," Phys. Rev. Lett. 110, 206802 (2013).

20. S. Schietinger, M. Barth, T. Aichele, and O. Benson, "Plasmon-Enhanced Single Photon Emission from a Nanoassembled Metal-Diamond Hybrid Structure at Room Temperature," Nano Lett. 9, 1694 (2009).

21. T. B. Hoang, G. M. Akselrod, and M. H. Mikkelsen, "Ultrafast RoomTemperature Single Photon Emission from Quantum Dots Coupled to Plasmonic Nanocavities," Nano Lett. 16, 270 (2016).

22. C. Van Vlack, Philip Trøst Kristensen, and S. Hughes, "Spontaneous emission spectra and quantum light-matter interactions from a strongly coupled quantum dot metal-nanoparticle system," Phys. Rev. B 85 075303 (2012).

23. A. Archambault, F. Marquier, J.-J. Greffet, and C. Arnold, "Quantum theory of spontaneous and stimulated emission of surface plasmons," Phys. Rev. B 82, 035411 (2010).

24. I. Bulu, T. Babinec, B. Hausmann, J. T. Choy, and M. Loncar, "How to deal with the loss in plasmonics and metamaterials," Opt. Express, 19, 5268 (2011).

25. J. B. Khurgin, "Replacing noble metals with alternative materials in plasmonics and metamaterials: how good an idea?," Phil. Trans. R. Soc. A 375, 20160068 (2017)

26. F. S. Johnson, "Physical cause of group velocity in normally dispersive, nondissipative media," Am. J. Phys. 58, 1044 (1990)

27. J. B. Khurgin, "Relative merits of phononics vs. plasmonics: the energy balance approach," Nanophotonics 7, 305 (2018).

28. A. Rodriguez, M. Soljačić, J. D. Joannopoulos, and S. G. Johnson, “ $\chi^{(2)}$ and $\chi^{(3)}$ harmonic generation at a critical power in inhomogeneous doubly resonant cavities," Opt. Express 15, 7303 (2007).

29. P. B. Johnson and R. W. Christy, "Optical Constants of the Noble Metals," Phys. Rev. B 64370 (1972)

30. L. D. Landau, L. P. Pitaevskii, and E. M. Lifshitz, Electrodynamics of Continuum Media, Second Edition: Volume 8 (Course of Theoretical Physics) (Butterworth-Heinemann, Amsterdam, 1984).

31. Y. Jiang and M. Liu, "Electromagnetic force in dispersive and transparent media," Phys. Rev. E 58, 6685 (1998).

32. G. Sun and J. B. Khurgin, Plasmonics and Plasmonic Metamaterials (World Scientific, Singapore, 2011).

33. D. Brissinger, L. Salomon, and F. De Fornel, “Unguided plasmon-mode resonance in optically excited thin film: exact modal description of Kretschmann-Raether experiment," J. Opt. Soc. Am. B 30, 333 (2013).

34. M. O. Scully, and M. S. Zubairy, Quantum Optics (Cambridge University Press, 1997).

35. J. Vučković, D. Englund, D. Fattal, E. Waks, and Y. Yamamoto, "Generation and manipulation of nonclassical light using photonic crystals," Physica E 32, 466 (2006)
書式変更：フォントの色：自動

書式変更：フォントの色：自動 
36. H. Iwase, "Polarized spontaneous emission from an emitter in controlled nodal vacuum fluctuations near a single high reflector," J. Opt. Soc. Am. B 31, 237 (2014).

37. A. Raman and S. Fan, "Photonic Band Structure of Dispersive Metamaterials Formulated as a Hermitian Eigenvalue Problem," Phys. Rev. Lett. 104, 087401 (2010)

38. J. D. Joannopoulos, R. D. Meade, and J. N.Winn, Photonic Crystals: Molding the Flow of Light (Princeton Univ. Press, 1995).

39. G. Bernasconi, J. Butet, and O. J. F. Martin, "Mode analysis of secondharmonic generation in plasmonic nanostructures," J. Opt. Soc. Am. B 33, 768 (2016)

40. A. L. Lereu, M. Zerrad, A. Passian, and C. Amra, "Surface plasmons and Bloch surface waves: Towards optimized ultra-sensitive optical sensors," Appl. Phys. Lett. 111, 011107 (2017).

41. Y. Xu, R. K. Lee, and A. Yariv, "Propagation and second-harmonic generation of electromagnetic waves in a coupled-resonator optical waveguide," J. Opt. Soc. Am. B 17, 387 (2000).

42. M. Bagherian, S. Kouchekian, I. Rothstein, and A. Passian, "Quantization of surface charge density on hyperboloidal and paraboloidal domains with application to plasmon decay rate on nanoprobes," Phys. Rev. B 98, 125413 (2018).

43. E.A. Matute, "On the vector solutions of Maxwell equations in spherica coordinate systems," REVISTA MEXICANA DE FI'SICA E 51, 31 (2005).

44. H. Bücher, K. H. Drexhage, M. Fleck, H. Kuhn, D. Möbius, F. P. Schäfer, Sondermann, W. Sperling, P. Tillmann and J. Wiegand, "Controlled Transfer of Excitation Energy Through Thin Layers," Mol. Cryst. 2, 199 (1967). 